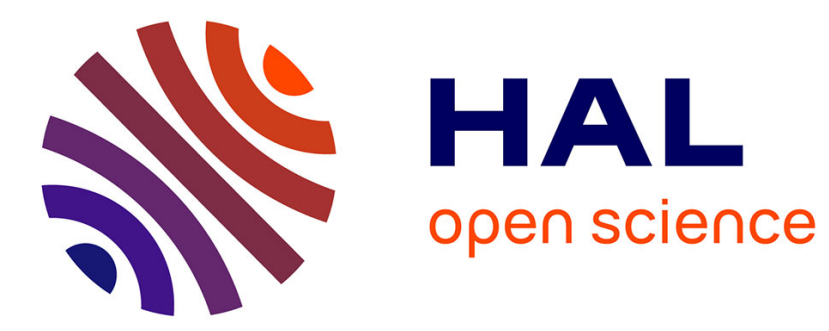

\title{
The Normal Modes of Nonlinear n-Degree-of-Freedom Systems
}

Reinhardt Rosenberg

\section{To cite this version:}

Reinhardt Rosenberg. The Normal Modes of Nonlinear n-Degree-of-Freedom Systems. Journal of Applied Mechanics, 1962, 29 (1), pp.7-14. 10.1115/1.3636501 . hal-01344457

\section{HAL Id: hal-01344457 \\ https://hal.science/hal-01344457}

Submitted on 12 Jul 2016

HAL is a multi-disciplinary open access archive for the deposit and dissemination of scientific research documents, whether they are published or not. The documents may come from teaching and research institutions in France or abroad, or from public or private research centers.
L'archive ouverte pluridisciplinaire $\mathbf{H A L}$, est destinée au dépôt et à la diffusion de documents scientifiques de niveau recherche, publiés ou non, émanant des établissements d'enseignement et de recherche français ou étrangers, des laboratoires publics ou privés. 


\section{The Normal Modes of Nonlinear n-Degree-of-Freedom Systems}

A system of $n$ masses, equal or not, interconnected by nonlinear "symmetric" springs, and having $n$ degrees of freedom is examined. The concept of normal modes is rigorously defined and the problem of finding them is reduced to a geometrical maximumminimum problem in an $n$-space of known metric. The solution of the geometrical problem reduces the coupled equations of motion to $n$ uncoupled equations whose natural frequencies can always be found by a single quadrature. An infinite class of systems, of which the linear system is a member, has been isolated for which the frequency amplitude can be found in closed form.

In some earlier papers $[1,2,3]^{1}$ the "normal modes" of certain nonlinear two-degree-of-freedom systems were discussed. These systems consisted of masses and springs, a restriction being that the forces with which the springs resist being deflected are odd functions of these deflections.

Evidently, these papers have dealt with a heretofore undefined subject matter since the concept of normal modes is generally defined for linear systems only. In them, "normal solutions" is the term associated with a fundamental set of solutions, and it is a well-known property of these, that a linear combination of the normal solutions yields all solutions of the system. Even if it should be possible to isolate, for nonlinear systems, solutions which are equivalent to the normal vibrations of linear systems, such solutions cannot be used to construct new solutions, since the superposition principle is ipso facto inadmissible in nonlinear systems.

The earlier method [2] consisted in identifying the search for the eigenvectors with the solution of a maximum-minimum problem in two-space. Once the eigenvectors had been found in this manner they could be utilized to transform the two-degree-offreedom problem into two separate problems, each in a single degree of freedom. This approach suggested itself readily, and it was relatively simple to exploit it, because the geometrical problem (which is the equivalent of the dynamical one) is one of geometry in two dimensions. It is natural to inquire whether it is even possible, and if 80 , whether it is useful to extend this theory to $n$-dimensions. In fact, doubt as to the feasibility of this extension has formed the substance of a discussion of an earlier paper [3].2 It is the purpose of this paper to extend the earlier results to systems having $n$-degrees of freedom.

\section{Equations of Motion}

Consider a system of $n$-masses (either equal to or different from each other) interconnected by nonlinear springs, and the first and last of the masses connected by nonlinear springs to fixed points (or to infinitely large masses). The springs also may be equal to or different from each other. The system has $n$ degrees of freedom and is illustrated in Fig. 1. The spring force of each spring is an odd function of the spring deflection. Thus, if a spring $S_{i}$ is deflected by an amount $u_{i}$, it resists this defection with a force $S_{i}\left(u_{i}\right)$, and

$$
S_{i}\left(-u_{i}\right)=-S_{i}\left(u_{i}\right)
$$

There exists (by definition of admissible systems) an equilibrium position of the system, and the displacement of the $i$ th mass from its equilibrium position is denoted by $x_{i}$, so that the $x_{i},(i=[1,2$, $\ldots, n$ ) are the co-ordinates of the problem. Then, the equations of motion of the system are

$$
\left.m_{i} \ddot{x}_{i}=S_{i}\left(x_{i-1}-x_{i}\right)-S_{i+1}\left(x_{i}-x_{i+1}\right), \begin{array}{rl}
i & =1,2, \ldots, n \\
x_{0} & =x_{n+1} \equiv 0
\end{array}\right\}
$$

In what follows, we shall only admit springs whose forces are everywhere analytic in the deflections and, moreover, we shall assume that these forces are represented with a satisfactory degree of accuracy by finite Taylor expansions. In the technical sense, these restrictions represent no limitations on the springs. Under them, the equations of motion become

$$
\begin{aligned}
& m_{i}{\ddot{x_{i}}}_{i}=\sum_{j=1,3, \ldots}^{r_{3}} a_{i, j}\left(x_{i-1}-x_{i}\right)^{i} \\
& \left.-\sum_{j=1,3, \ldots}^{r_{i+1}} a_{i+1, i}\left(x_{i}-x_{i+1}\right)^{i}, \begin{array}{rl}
i & =1,2, \ldots, n \\
x_{0} & =x_{n+1} \equiv 0
\end{array}\right\}
\end{aligned}
$$

Finally, it is convenient to normalize the co-ordinates by means of the transformations

$$
\xi_{i}=m_{i}{ }^{1 / 2} x_{i}, \quad i=1,2, \ldots, n
$$

The equations of motion then take on their final form

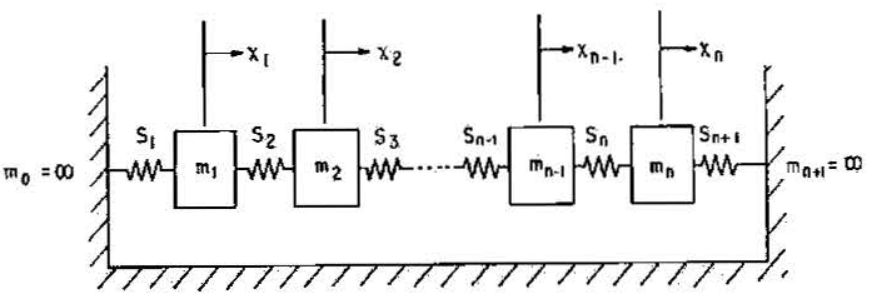

Fig. 1 


$$
\begin{aligned}
\ddot{\xi}_{i} & =\sum_{j=1,3, \ldots}^{r_{i}}\left\{\frac{a_{i, j}}{m_{i}^{1 / 2}}\left(\frac{\xi_{i-1}}{m_{i-1}{ }^{1 / 2}}-\frac{\xi_{i}}{m_{i}^{1 / 2}}\right)^{j}\right\} \\
- & \sum_{j=1,3, \ldots}^{r_{i+1}}\left\{\frac{a_{i+1, i}}{m_{i}^{1 / 2}}\left(\frac{\xi_{i}}{m_{i}^{1 / 2}}-\frac{\xi_{i+1}}{m_{i+1}{ }^{1 / 2}}\right)^{\prime}\right\} \quad \begin{aligned}
i & =1,2, \ldots, n \\
m_{0} & =m_{n+1}=\infty
\end{aligned}
\end{aligned}
$$

Since the system is conservative, the right-hand sides of the equations of motion must be derivable from a potential function. In fact, one finds readily

$$
\begin{gathered}
\xi_{i}=\frac{\partial U}{\partial \xi_{i}}, \quad i=1,2, \ldots, n \\
U=-\sum_{i=1,2}^{n+1} \ldots \sum_{j=1,3, \ldots}^{r_{i}}\left\{\frac{a_{i, j}}{j+1}\left(\frac{\xi_{i-1}}{m_{i-1}^{1 / 2}}-\frac{\xi_{i}}{m_{i}^{1 / 2}}\right)^{i+1}\right\} \\
m_{0}=m_{n+1}=\infty
\end{gathered}
$$

Evidently, $U$ is symmetric with respect to the origin; in other words, $U$ remains unchanged if any one, or some, or all of the $\xi_{i-1}$ are replaced by $-\xi_{i-1}$ provided that, at the same time, the corresponding $\xi_{i}$ are replaced by $-\xi_{i}$. This important property is a direct consequence of the fact that all spring forces are odd functions of the spring displacements.

The transformations ( 3 ) have normalized the system so that, in the transformed co-ordinates, the kinetic energy is

$$
2 T\left(\dot{\xi}_{i}\right)=\sum_{i}^{n} \dot{\xi}_{i}^{2}
$$

while, in the physical co-ordinates,

$$
2 T\left(x_{i}\right)=\sum_{i}^{n} m_{i} \dot{x}_{i}^{2}
$$

However, this transformation also admits a physical interpretation. The nontransformed equations (2) are those of the physical system of Fig. 1. However, the transformed equations of motion may be regarded as those of a unit mass which moves in an $n$-dimensional space under forces which are derivable from the potential function $U$ defined in $(6)$; the $\xi_{i}$ are, then, an orthogonal co-ordinate system in the $n$-space. Finally, it should be observed that the system (4), or (5) and (6), reduces to the linear problem when $j=1$.

\section{Normal Modes of Linear System}

When dealing with the linear system, the normal modes, or the eigenvectors, are usually determined after the eigenvalues are found. In fact, one usually substitutes sinusoidal functions of time

$$
\xi_{i}(t)=b_{i} \cos \omega t, \quad i=1,2, \ldots, n
$$

into the equations of motion and finds in this manner a characteristic function whose zeros are the eigenvalues. Then, the $r$ th eigenvector $v_{r}\left(\omega_{r}\right)$ is found by attaching to each eigenvalue $\omega_{r}$ the associated eigenvector $v_{r}$. The $b_{i r}$ are then the $n$-components of $v_{r}$. It is evident that, in the very first step, this method makes use of a property which is unique to the linear problem; namely, that the displacements are sinusoidal functions of time. Consequently, this method can never lead to a generalization of the concept of normal modes which comprises the nonlinear problem, and which reduces to the familiar meaning of the eigenvector when the problem reduces to the linear one.

Here, we wish to find a new way of defining normal modes of the linear system which mugt be such that $(a)$ the normal modes of the linear system are uniquely and correctly defined by it and (b) the definition must be capable of a simple extension to the nonlinear system.

This definition begins with the observation easily deducible from (7), that

$$
\left.\begin{array}{rl}
\xi_{i}(t) & =\xi_{i}(t+T) \\
\frac{\xi_{i+1}(t)}{\xi_{1}(t)} \equiv \frac{b_{i+1}}{b_{1}}=c_{i+1}
\end{array}\right\} \begin{aligned}
& i=1,2, \ldots, n \\
& \xi_{n+1} \equiv 0
\end{aligned}
$$

The first of these states that the normal solutions of the linear system are all periodic of the same period, while the second states that the ratio of the displacement of any one mass to that of any other is identically equal to a constant for all time. The first is a property which must be retained in the nonlinear case while the second may either be retained in the form

$$
\xi_{i+1}=c_{i+1} \xi_{1}, \quad \begin{aligned}
& i=1,2, \ldots, n \\
& \xi_{n+1} \equiv 0
\end{aligned}
$$

or, it is capable of the obvious generalization

$$
\xi_{i+1}=\xi_{i+1}\left(\xi_{1}\right), \quad \begin{aligned}
& i=1,2, \ldots, n \\
& \xi_{n+1} \equiv 0
\end{aligned}
$$

where the $\xi_{i+1}$ are single-valued functions of $\xi_{1}$.

The second of (8) harbors a well-known property of normal vibrations. Suppose there exists a time $t_{0}$ such that $\xi_{1}\left(t_{0}\right)=0$. Then, it must be true, in view of the second of (8), that $\xi_{2}, \xi_{3}, \ldots$ $\xi_{n}$ all vanish at the same instant. In other words, all masses of the system of Fig. 1 pass through their equilibrium position at the same instant if the system vibrates in normal modes. The two properties of vibrations in normal modes, (a) that all masses vibrate at the same frequency and, (b) that they pass through their equilibrium position at the same time is, in fact, a description of normal vibrations. If these properties are to be retained in the nonlinear system as generalized in (10), the boundary conditions

$$
\xi_{i+1}(0)=0, \quad i=1,2, \ldots, n-1
$$

must be added to $(10)$. If, however, $(9)$ is to be retained, the condition (11) is automatically satisfied.

One additional property of the normal modes of linear systems must be exhibited before the array is sufficient for actually finding the eigenvectors; this property is connected with the manner in which the system is set into motion. Let us suppose that we start the motion without initial velocity, so that the initial conditions are

$$
\xi_{i}(0)=b_{i}, \quad \xi_{i}(0)=0, \quad i=1,2, \ldots, n
$$

[We note here that the $b_{i}$ are not arbitrary. In fact, with arbitrary values of $b_{i}$ the system would not vibrate in normal modes; instead, energy exchange between the masses (or in the transformed system Lissajoux figures of the unit mass) would result. In fact, the $b_{i}$ are the $\xi_{i}$-components of an eigenvector; finding these is the essential problem in the solution of the eigenvalue problem. However, we assume here a priori the well-known fact that initial conditions like (9), and resulting in normal vibrations do exist. If it should be found in the generalization to nonlinear systems that such initial conditions do not exist, we shall say that the nonlinear system is not capable of vibrating in normal modes.]

It follows from an application of L'Hopital's rule that

$$
\frac{\xi_{i+1}(0)}{\xi_{1}(0)}=\frac{\ddot{\xi}_{i+1}(0)}{\ddot{\xi}_{1}(0)}=\frac{\partial U / \partial \xi_{i+1}}{\partial U / \partial \xi_{2}}, \quad \begin{aligned}
& i=1,2, \ldots, n \\
& \xi_{n+1} \equiv 0
\end{aligned}
$$

or, at the time $t=0$, 


$$
\frac{d \xi_{1}}{\partial U / \partial \xi_{1}}=\frac{d \xi_{2}}{\partial U / \partial \xi_{2}}=\ldots=\frac{d \xi_{n}}{\partial U / \partial \xi_{n}}
$$

The meaning of this important property will be discussed a little further on. All these properties of normal modes of linear systems are easily interpreted in a geometrical manner.

Consider the $(n+1)$-dimensional space whose orthogonal coordinates are $\xi_{1}, \xi_{2}, \ldots, \xi_{n}, U$. When the system is released at the time $t=0$, it occupies a position of maximum potential

$$
U=-U_{0}
$$

because it is released without initial velocity. But $U=-U_{0}$ defines an $n$-dimensional equipotential surface or, more precisely, it defines the projection of the equipotential surface $U=-U_{0}$ on the $\left(\xi_{1}, \xi_{2}, \ldots, \xi_{n}\right)$-space. In fact, (14) defines a domain in the $n$-space surrounding the origin, and all solutions must lie in this domain (for, if they did not, the potential would have to exceed its maximum value $-U_{0}$ ). We shall, therefore, call it the "bounding surface."

Now, in virtue of the second of (8), the normal modes satisfy the relations

$$
\frac{\xi_{2}(t)}{\xi_{1}(t)} \equiv c_{2}, \quad \frac{\xi_{3}(t)}{\xi_{1}(t)} \equiv c_{3}, \ldots, \quad \frac{\xi_{n}(t)}{\xi_{1}(t)} \equiv c_{n}
$$

The first of these, i.e., $\xi_{3}=c_{2} \xi_{1}$, robs the $n$-space of one dimension (because one of the co-ordinates is expressible in terms of another). In fact, it defines an $(n-1)$-dimensional hyperspace which intersects the $\xi_{1} \xi_{2}$-plane on the line $\xi_{2}=c_{2} \xi_{1}$. In this hyperspace, the equation $\xi_{3}=c_{2} \xi_{1}$ defines an $(n-2)$-dimensional hyperspace which intersects the $\xi_{1} \xi_{3}$-plane on the line $\xi_{8}=c_{3} \xi_{1}$. A continuation of this argument finally defines a straight line passing through the origin of the $n$-space and intersecting at least twice the bounding surface within which the solutions must lie. The component of this straight line along the $\xi_{1}$-axis, say, is $b_{i} \xi_{i}$. We may picture the normal vibration as a periodic (and in the linear problem a simple harmonic) motion of the unit mass traveling along this line between the points where that line pierces the surface defined by $U=-U_{0}$. The projections of that motion on the $\xi_{i}$-axes, $(i=1,2, \ldots, n)$ are the normal vibrations of the system.

We are now in a position to discuss the meaning of (13). At the time $t=0$, the point moving along the straight line defined previously, and termed a "modal relation" [2], lies on the bounding surface, or in the domain defined by (14). At the same instant, the relations (13) apply. But, since from (13)

$$
d U=\sum_{i=1,2, \ldots}^{n} \frac{\partial U}{\partial \xi_{i}} d \xi_{i}=0
$$

It is evident that (13) are merely the well-known expressions for the conditions that the modal line intersects the bounding surface defined by $U=-U_{0}$ orthogonally.

We have now collected the properties, necessary to define a normal mode of the linear system having $n$ degrees of freedom.

Definition. A normal mode of the linear system

$$
\ddot{\xi}_{i}=\frac{\partial U}{\partial \xi_{i}}, \quad i=1,2, \ldots, n
$$

where $U$ is given by (6) with $j=1$, it is a straight line in the ( $\xi_{1}$, $\left.\xi_{2}, \ldots, \xi_{s}\right)$-space defined by the $(n-1)$ equations

$$
\xi_{i}=c_{i} \xi_{1}, \quad i=2,3, \ldots, n
$$

which are satisfied for all $t$ by periodic solutions $\xi_{i}(t)=\xi_{i}(t+T)$ of (16) in the closed domain $U=-U_{0}$ of the $\left(\xi_{1} \xi_{2}, \ldots, \xi_{n}\right)$-space, which passes through the origin of that space and which intersects the bounding surface defined by $U=-U_{0}$ orthogonally.
An Example. As an example of the foregoing definition, consider the three-dimensional linear problem, and let

$$
\begin{aligned}
& x_{1}=x, \quad x_{2}=y, \quad x_{3}=z, \\
& \xi_{1}=\xi, \quad \xi_{2}=\eta, \quad \xi_{3}=\zeta
\end{aligned}
$$

The equations of motion of the physical system are

$$
\begin{aligned}
& m_{1} \ddot{x}=-a_{1} x-a_{3}(x-y) \\
& m_{2} \ddot{y}=a_{2}(x-y)-a_{3}(y-z) \\
& m_{3} \ddot{z}=a_{3}(y-z)-a_{4} z
\end{aligned}
$$

The transformed equations are

$$
\begin{aligned}
& \ddot{\xi}=-\frac{a_{1}}{m_{1}^{1 / 2}}\left(\frac{\xi}{m_{1}^{1 / 2}}\right)-\frac{a_{2}}{m_{1}^{1 / 2}}\left(\frac{\xi}{m_{3}^{1 / 2}}-\frac{\eta}{m_{2}^{1 / 2}}\right) \\
& \ddot{\eta}=+\frac{a_{2}}{m_{2}^{1 / 2}}\left(\frac{\xi}{m_{1}^{1 / 2}}-\frac{\eta}{m_{2}^{1 / 2}}\right)-\frac{a_{3}}{m_{2}^{1 / 2}}\left(\frac{\eta}{m_{2}^{1 / 2}}+\frac{\zeta}{m_{3}^{1 / 2}}\right) \\
& \dot{\zeta}=+\frac{a_{3}}{m_{3}^{1 / 2}}\left(\frac{\eta}{m_{2}^{1 / 2}}-\frac{\zeta}{m_{3}^{1 / 2}}\right)-\frac{a_{4}}{m_{3}^{1 / 2}}\left(\frac{\zeta}{m_{3}^{1 / 2}}\right)
\end{aligned}
$$

We may also write the system as

$$
\ddot{\xi}=\frac{\partial U}{\partial \xi}, \quad \ddot{\eta}=\frac{\partial U}{\partial \eta}, \quad \xi=\frac{\partial U}{\partial \zeta}
$$

where the potential function is defined as

$$
\begin{aligned}
U=-\frac{a_{1}}{2}\left(\frac{\xi}{m_{1}{ }^{1 / 2}}\right)^{2} & -\frac{a_{2}}{2}\left(\frac{\xi}{m_{1}^{1 / 2}}-\frac{\eta}{m_{2}^{1 / 2}}\right)^{2} \\
& -\frac{a_{3}}{2}\left(\frac{\eta}{m_{2}^{1 / 2}}-\frac{\zeta}{m_{3}^{1 / 2}}\right)^{2}-\frac{a_{4}}{2}\left(\frac{\zeta}{m_{3}^{1 / 2}}\right)^{2}
\end{aligned}
$$

It is evident that

$$
U=-U_{0}
$$

defines an ellipsoid in the $(\xi, \eta, \zeta)$-space which is symmetric with respect to the origin.

Let the normal solutions be

$$
\left.\begin{array}{c}
\frac{\eta(t)}{\xi(t)}=c_{\eta}, \quad \frac{\zeta(t)}{\xi(t)}=c_{\zeta} \\
\text { or } \\
\eta=c_{\eta} \xi, \quad \zeta=c_{\zeta} \xi
\end{array}\right\} c_{\eta, \zeta \text { are constants }}
$$

The first of these defines a plane in the $(\xi, \eta, \zeta)$-space which contains the $\zeta$-axis, and which intersects the $(\xi, \eta)$-plane on the line $\eta=c_{\eta} \xi$. The second defines another plane which contains the $\eta$-axis, and which intersects the $(\xi, \zeta)$-plane on the line $\zeta=$ $c_{5} \xi$. The intersection of these two planes is the line $(O P)$, as shown in Fig. 2, and that line is the modal relation. Moreover,
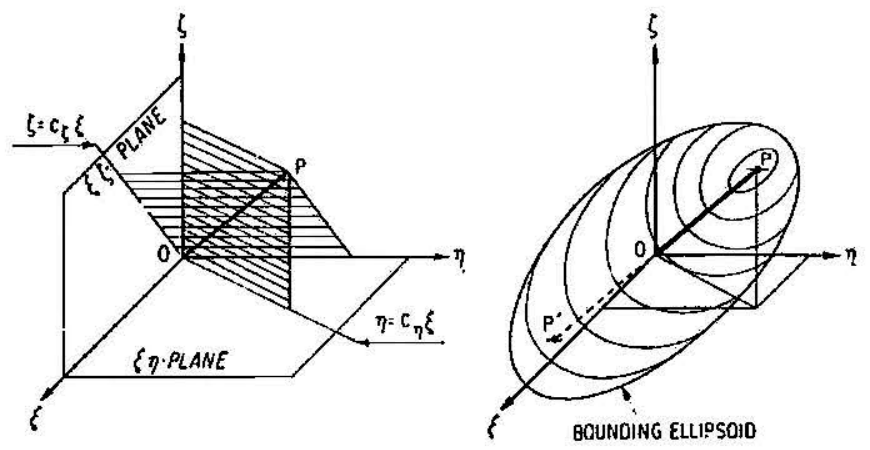

Fig. 2 
that line intersects the ellipsoid $U=-U_{0}$ at two points $P$ and $P^{\prime}$ and, at these points, the modal line is normal to the surface of the ellipsoid.

The model of the motion in hand is that of the unit mass traveling along the modal line in simple harmonic motion between the points $P$ and $P^{\prime}$ where the modal line intersects the surface of the ellipsoid. At the extremities, the velocity vanishes, and the co-ordinates of the point $P$ are the initial displacement components.

\section{Generalization of Normal Modes}

In a purely verbal manner we shall say that the nonlinear system in Fig. 1 vibrates in normal modes when all masses execute periodic motions of the same period, when all of them pass through the equilibrium position at the same instant, and when, at any time $t$, the position of all the masses is uniquely defined by the position of any one of them. In view of these properties and of the preceding section, we give for normal modes, in general, the following:

Definition. The $m$ th normal mode of the system

$$
\ddot{\xi}_{i}=\frac{\partial U}{\partial \xi_{i}}, \quad i=1,2, \ldots, n
$$

where $U$ is given by (6) is a line (curved or straight) in the $\left(\xi_{1}, \xi_{2}, \ldots, \xi_{n}\right)$-space which is defined by the $(n-1)$ singlevaluzed functions

$$
\xi_{i}=\xi_{i m}\left(\xi_{1}\right), \quad i=2,3, \ldots, n
$$

which are satisfied for all $t$ by the periodic solutions $\xi_{i}(t)=\xi_{i}(t$ $+T)$ of (18) in the closed domain $U=-U_{0}$ of the $\left(\xi_{1}, \xi_{\xi_{1}}, \ldots, \xi_{n}\right)-$ space and which satisfies the boundary conditions that:

(a) All $\xi_{\text {im }}(0)=0$, or the line passes through the origin of the $\left(\xi_{1}, \xi_{2}, \ldots, \xi_{n}\right)$-space.

(b) The line intersects the bounding surface defined by $U=$ $-U_{0}$ orthogonally.

An illustration corresponding to that of Fig. 2 but applied to the nonlinear system of three degrees of freedom is shown in Fig. 3. It should be abserved that, in the linear case, the bounding surface is an ellipsoid while, in the nonlinear case it is an ovaloid which is, in general, not an ellipsoid.

While only one modal relation is referred to in the definitions and only one has been illustrated in Figs. 2 and 3, it is known that, in the linear case at least, as many such modal lines exist as there are degrees of freedom. For instance, in the example of the threedegree-of-freedom system shown in Fig. 2, three modal lines exist and they are, in fact, the principal axes of the ellipsoid.

\section{The Decoupled Equations}

For the present, we leave open the question as to whether, or how, the modal lines can be found. Supposing simply that they
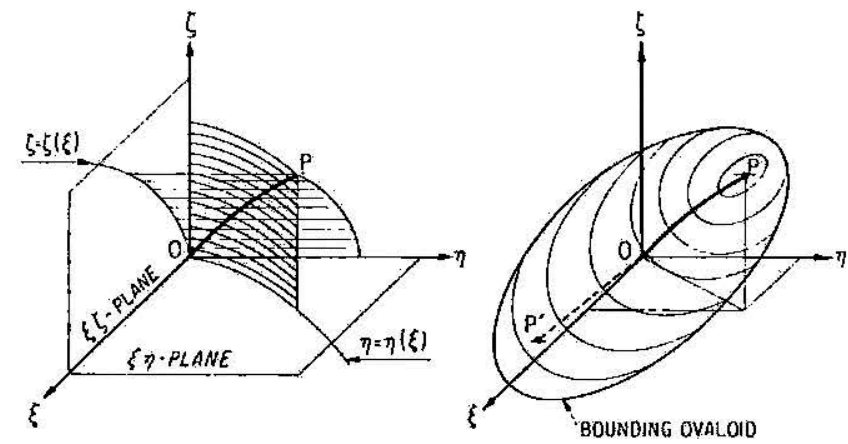

Fig. 3 are known, they can be used in an obvious way to reduce the $n$-degree-of-freedom system of $n$-equations, each in a single degree of freedom. For instance, the $r$ th equation of motion is

$$
\ddot{\xi}_{r}=\frac{\partial U}{\partial \xi_{r}}\left(\xi_{r-1}, \xi_{r r} \xi_{r+1}\right)
$$

in accordance with (5). In (20), the expression on the right-hand side denotes the fact that, in general, the derivative $\partial U / \partial \xi_{\text {r }}$ depends on the three variables $\xi_{r-1}, \xi_{r}, \xi_{r+1}$. If the equations (19) in one of the modes are known, one knows in particular the functions

$$
\begin{aligned}
\xi_{r-1} & =\xi_{r-1}\left(\xi_{1}\right) \\
\xi_{r} & =\xi_{r}\left(\xi_{1}\right) \\
\xi_{r+1} & =\xi_{r+1}\left(\xi_{1}\right)
\end{aligned}
$$

Introducing them into $(20)$ gives

$$
\xi_{r}^{\prime} \ddot{\xi}_{1}+\xi_{r}^{\prime \prime} \xi_{1}^{2}=\frac{\partial U}{\partial \xi_{r}}\left(\xi_{r-1}\left(\xi_{1}\right), \xi_{r}\left(\xi_{1}\right), \xi_{r+1}\left(\xi_{1}\right)\right)
$$

where primes denote differentiation with respect to $\xi_{1}$, and dots differentiation with respect to time $t$. It follows that (21) is au equation in the single dependent variable $\xi_{1}$. If there are $n$ modes $r=1,2, \ldots, n$, the system has now been reduced to $n$ equations, each in the variable $\xi_{1}$.

A simplification of (21) can be achieved if we choose $r=1$ (which we are free to do). In that case, (17) is

$$
\ddot{\xi}_{1}=\frac{\partial U}{\partial \xi_{1}}\left(\xi_{1}, \xi_{2}\right)
$$

Knowledge of the equations defining the modal relation includes the equation

$$
\xi_{2}=\xi_{2}\left(\xi_{1}\right)
$$

Then, (19) becomes simply

$$
\ddot{\xi}_{1}=\frac{\partial U}{\partial \xi_{1}}\left(\xi_{1}, \xi_{2}\left(\xi_{1}\right)\right)
$$

which is, obviously, an equation in $\xi_{1}$, only. If there are $n$ modes, there are $n$ equations (23) and, introducing each in turn into (22), there are $n$ equations of the form (24), all in $\xi_{1}$.

The application of this procedure to the linear three-degree-offreedom system may be instructive. The first equation of motion is

$$
\dddot{\xi}=-\frac{a_{1}}{m_{1}^{1 / 2}}\left(\frac{\xi}{m_{1}^{1 / 2}}\right)-\frac{a_{2}}{m_{1}^{1 / 2}}\left(\frac{\xi}{m_{1}^{1 / 2}}-\frac{\eta}{m_{2}^{1 / 2}}\right)
$$

and the equations of the modal relations are supposed known. But this implies knowledge of the three constants $c_{\eta r}$ in

$$
\eta=c_{\eta r} \xi, \quad r=1,2,3
$$

Intruducing this relation into the first equation of motion yields the three equations

$$
\ddot{\xi}=-\frac{a_{1}}{m_{1}}\left\{1-\frac{a_{2}}{a_{1}}\left[1-\left(\frac{m_{1}}{m_{2}}\right)^{1 / 2} c_{\eta r}\right]\right\} \xi, \quad r=1,2,3
$$

From these, the three natural frequencies, or eigenvalues, can now be found without difficulty.

It should be noticed that knowledge of the eigenvectors has been assumed without any indication as to how they can be found. We shall now discuss the question of finding the eigenvectors without prior knowledge of the eigenvalues. 


\section{Determination of the Eigenvectors}

The dynamical problem in hand is to find the normal solutions of the system

$$
\ddot{\xi}_{i}=\frac{\partial U}{\partial \xi_{i}}, \quad i=1,2, \ldots, n
$$

Any solution of this system (including the normal solutions) is of the form

$$
\xi_{i}=\xi_{i}(t), \quad i=1,2, \ldots, n
$$

Elimination of time between these functions and regarding $\xi_{1}$ (for instance) as the independent variable, transforms (26) into

$$
\xi_{i}=\xi_{i}\left(\xi_{1}\right), \quad i=2,3, \ldots, n
$$

These functions constitute the trajectory of the system in the $\left(\xi_{1}, \xi_{2}, \ldots, \xi_{n}\right)$-space. In a physical sense, they represent the trajectory of the unit mass of the system (4), or (5) and (6). It is well known [4] that this trajectory is the geodesic in an $n$-dimensional space whose metric is

$$
d \sigma^{2}=\left(U_{0}+U\left(\xi_{1}, \xi_{2}, \ldots, \xi_{n}\right)\right) \sum_{j=1,2}^{n} \ldots \xi_{i}{ }^{2}
$$

Consequently, the differential equations of this geodesic are the Euler equations of the variational problem

$$
\int_{\xi_{10}}^{\xi_{11}}\left(U_{0}+U\right)^{1 / 2}\left(1+\sum_{j=2,3, \ldots}^{n} \xi_{j}{ }^{2}\right)^{1 / 2} d \xi_{1}=\text { stationary }
$$

These Euler equations are

$$
\begin{aligned}
& 2\left(U_{0}+U\right)\left\{\xi_{i}^{\prime \prime}\left(1+\sum_{j \neq i}^{n} \xi_{i}^{\prime 2}\right)-\sum_{j \neq i}^{n} \xi_{i}^{\prime} \xi_{i}^{\prime} \xi_{j}^{\prime \prime}\right\} \\
&+\left(1+\sum_{j}^{n} \xi_{i}^{\prime 2}\right)\left\{\xi_{i}^{\prime} \frac{\partial U}{\partial \xi_{i}}+\sum_{j \neq i}^{n} \xi_{j}^{\prime} \xi_{i}{ }^{\prime} \frac{\partial U}{\partial \xi_{j}}\right. \\
&\left.-\left(1+\sum_{j \neq i}^{n} \xi_{i}^{\prime 2}\right) \frac{\partial U}{\partial \xi_{i}}\right\}=0 \\
& i, j=2,3, \ldots, n
\end{aligned}
$$

While every solution of (30) represents a trajectory of the dynamical system in the $\left(\xi_{1}, \xi_{2}, \ldots, \xi_{n}\right)$-space, not all are modal lines. The modal lines are those solutions of (28) which satisfy the boundary conditions listed in the definition of normal modes. Since (30) is a system of $(n-1)$ second-order equations, it gives rise to $2(n-1)$ constants of integration. But the trajectory is defined by the $(n-1)$ equations $(27)$, and the satisfaction of the required boundary conditions (one being the value at the origin, the other the value of the slope on the bounding surface) requires the determination of $2(n-1)$ constants. Therefore, it is possible, in principle at least, to solve the problem. Nevertheless, the system (30) is such a complicated system of highly nonlinear differential equations that the prospect of finding the general solution may be confidently regarded as hopeless.

\section{Straight Modal Relations}

Let us assume that, for certain forms of $U$, the modal relations are straight lines. If they are, the second derivatives $\xi_{i}{ }^{\prime \prime},(j=$ $2,3, \ldots, n)$ vanish. Then, since

$$
1+\sum_{j}^{n} \xi_{i}{ }^{2}>0
$$

these straight modal relations must satisfy the system of equations

$$
\begin{aligned}
\xi_{1}^{\prime} \frac{\partial U}{\partial \xi_{i}}+\sum_{j \neq i}^{n} \xi_{j}^{\prime} \xi_{i}^{\prime} \frac{\partial U}{\partial \xi_{i}} \\
=\left(1+\sum_{j \neq i .}^{n} \xi_{j}^{\prime 2}\right) \frac{\partial U}{\partial \xi_{i}} ; \quad i, j=2,3, \ldots, n
\end{aligned}
$$

Writing (31) in terms of differentials (instead of derivatives) and adding $\left(\partial U / \partial \xi_{i}\right) d \xi_{i}{ }^{2}$ to both sides of the equation, it is found that

$$
\frac{d \xi_{1}}{\partial U / \partial \xi_{1}}=\frac{d \xi_{2}}{\partial U / \partial \xi_{2}}=\ldots=\frac{d \xi_{n}}{\partial U / \partial \xi_{n}}
$$

[Actually, proeeeding in the manner indicated yields (32) with

$$
\frac{d \xi_{1}}{\partial U / \partial \xi_{1}}
$$

absent. However, $\xi_{1}$ is not intrinsically a preferred co-ordinate; it has assumed a special position only because we have assigned to it the role of the independent variable. Thus it is clear that the first term in (32) may be added to the set. One can also obtain this result in a formal manner by considering all $\xi_{i}$ to depend on a parameter $\alpha_{\text {, }}$ and by treating the variational problem

$$
\int_{\alpha_{0}}^{\alpha_{1}}\left(U_{0}+U\right)^{1 / 2}\left(\sum_{j=1}^{n} \xi_{1,2}{ }^{2 \prime}\right)^{1 / 2} d \alpha=\text { stationary }
$$

where primes denote differentiation with respect to $\alpha$.$] But$ (32) is similar to (13), the only difference being that (13) was valid only at $t=0$ while (32) applies at every value of $t$. Therefore (32) implies that the modal line under discussion is normal to all equipotential surfaces

$$
U=-H \geqslant-U_{0}
$$

This leads to the following:

Theorem. Every straight line in the $\left(\xi_{1}, \xi_{2}, \ldots, \xi_{n}\right)$-space which intersects all equipotential surfaces orthogonally is a modal line.

Conversely, every modal line which is straight intersects all equipotential surfaces orthogonally.

\section{Homogeneous Systems}

Among the systems illustrated in Fig. 1 whose equations of motion are given by (4), we select the class whose equations of motion are

$$
\begin{aligned}
& \ddot{\xi}_{i}=\frac{a_{i, k}}{m_{\S}{ }^{1 / 2}}\left(\frac{\xi_{i-1}}{m_{i-1}{ }^{1 / 2}}-\frac{\xi_{i}}{m_{i}^{1 / 2}}\right)^{k} \\
& \left.-\frac{a_{i+1, k}}{m_{i}^{1 / 2}}\left(\frac{\xi_{i}}{m_{i}^{1 / 2}}-\frac{\xi_{i+1}}{m_{i+1}{ }^{1 / 2}}\right)^{k}, \begin{array}{l}
i=1,2, \ldots n \\
m_{0}=m_{n+1}=\infty \\
k=\text { odd integer }
\end{array}\right\}
\end{aligned}
$$

This class is called the homogeneous system of degree $k$ because the right-hand sides are homogeneous functions in the $\xi_{i}$ of degree $k$. We shall show that, for this class, the modal relations are straight.

The system (33) has considerable interest, both from the mathematical and from the physical point of view. Its mathematical interest resides in the fact that it represents a broad generalization of the linear problem since the linear system is one of its members. Moreover, as we shall show later, in homogeneous systems, the eigenvalues as functions of the amplitudes can be found in terms of tabulated functions and without the approximations that must usually be made in the treatment of nonlinear problems.

Physically, the homogeneous system is such that the springs 
resist being deflected with a force that is proportional to the $k$ th power of the deflection. Thus the system is of interest wherever springs are used which have this property.

For our purposes, it is more convenient to write (33) in the form

$$
\begin{gathered}
\ddot{\xi}_{i}=\frac{\partial U}{\partial \xi_{i}}, \quad i=1,2, \ldots, n \\
U=-\sum_{i}^{n+1} \frac{a_{i . k}}{k+1}\left(\frac{\xi_{i-1}}{m_{i-1}^{1 / 2}}-\frac{\xi_{i}}{m_{i}^{1 / 2}}\right)^{k+1}, \quad m_{0}=m_{n+1}=\infty
\end{gathered}
$$

We now make use of the following property of surfaces in Euclidian $n$-space': Consider a smooth surface in this space. Then, the straight line between the origin (of that space) and that point on the surface which is nearest to, or farthest from, the origin intersects the surface orthogonally.

We consider the equipotential surfaces

$$
\begin{aligned}
& U=-H=\text { const } \\
& 0>-H \geqslant-U_{0}
\end{aligned}
$$

and we seek those points on each of them whose distance from the origin is stationary. If the locus of these points is a straight line, its equation will not contain the distance of these points from the origin. Therefore the procedure is to write the equations of the equipotential surfaces in generalized polar co-ordinates and, then, to apply the condition which singles out those points on the surfaces whose distance from the origin is stationary. A necessary condition for the existence of straight modal relations is that the modules of the position vectors of these stationary points do not appear in the equations of the locus of these points. If the equations of the locus have real zeros, the conditions which are, both, necessary and sufficient for the existence of straight modal relations are fulfilled.

Let the generalized polar co-ordinates be

$$
\xi_{i}=r f_{i}\left(\theta_{1}, \theta_{1}, \ldots, \theta_{n-1}\right), \quad i=1,2, \ldots, n
$$

They are such that, when $r=1$ and the $\theta_{i}$ take on all possible values between $0<\theta_{i} \leqslant 2 \pi$, all points on a hypersphere of radius 1 in the $n$-space are defined. If these co-ordinates are introduced into the equations of the equipotential surfaces

$$
-U=\sum_{i=1}^{n+1} \frac{a_{i, k}}{k+1}\left(\frac{\xi_{i-1}}{m_{i-1}^{1 / 2}}-\frac{\xi_{i}}{m_{i}^{1 / 2}}\right)^{k+1}=H \leq U_{0}
$$

one finds

$$
-U=r^{k+1} \sum_{i=1,2, \ldots}^{n+1} \frac{a_{i, k}}{k+1}\left(\frac{f_{i-1}}{m_{i-1}^{1 / 2}}-\frac{f_{i}}{m_{i}^{1 / 2}}\right)^{k+1}=H
$$

or, more generally

$$
U\left(r, \theta_{1}, \theta_{4}, \ldots, \theta_{n-1}\right)=R(r) \theta\left(\theta_{1}, \theta_{2}, \ldots, \theta_{n-1}\right)=H
$$

Now, the locus of the stationary points in question is defined by

$$
d r=0
$$

which implies

$$
\frac{\partial U}{\partial \theta_{i}}=0, \quad i=1,2, \ldots, n-1
$$

In view of (36), the last equation is equivalent to

3 This is not the $n$-space whose metric is given in (26), The $n$ space under consideration here has the metric

$$
d \sigma^{2}=\sum_{i}^{n} d \xi_{i}^{2}
$$

$$
\frac{\partial \theta}{\partial \theta_{i}}=0, \quad i=1,2, \ldots, n-1
$$

and, thus, the condition which is necessary for the existence of straight modal relations is satisfied since $\theta$ is a function of the angles $\theta_{i}$ only. Whether the relations (37) have real roots $\theta_{i r}$, $(r=1,2, \ldots)$ cannot be answered without further caleulation because the $f_{i}$ have not been expressed explicitly as functions of the angles. We shall examine this question further for the case of the nonlinear three-degree-of-freedom system.

\section{Homogeneous System With Three Degrees of Freedom}

Consider a homogeneous system like that of Fig. 1 but having only three degrees of freedom, and let

$a_{1, k}=a_{1}, \quad a_{2, k}=a_{2}, \quad a_{3, k}=a_{3,} \quad a_{k, k}=a_{4} \quad \xi_{1}=\xi, \quad \xi_{2}=\eta$,

$$
\xi_{3}=\zeta, \quad \theta_{1}=\theta, \quad \theta_{2}=\varphi, \quad H=\bar{H}
$$

The equation of the equipotential surface is

$$
\begin{aligned}
-\frac{a_{1}}{k+1} & \left(\frac{\xi}{m_{1}^{1 / 2}}\right)^{k+1}+\frac{a_{2}}{k+1}\left(\frac{\xi}{m_{1}^{1 / 2}}-\frac{\eta}{m_{2}^{1 / 2}}\right)^{k+1} \\
& +\frac{a_{3}}{k+1}\left(\frac{\eta}{m_{2}^{1 / 2}}-\frac{\zeta}{m_{3}^{1 / 2}}\right)^{k+1}+\frac{a_{4}}{k+1}\left(\frac{\zeta}{m_{4}^{1 / 2}}\right)^{k+1}=F
\end{aligned}
$$

For simplicity (although this restrietion is not necessary) we assume that all masses are equal. Introducing the nomenclature

$$
\bar{H} \cdot m^{\frac{k+1}{2}}=H \text {, }
$$

the foregoing equation simplifies to

$$
\begin{aligned}
\frac{a_{1}}{k+1} \xi^{k+1}+\frac{a_{2}}{k+1}(\xi-\eta)^{k+1} & \\
& +\frac{a_{3}}{k+1}(\eta-\zeta)^{k+1}+\frac{a_{1}}{k+1} \zeta^{k+1}=H
\end{aligned}
$$

We now introduce polar co-ordinates

$$
\begin{aligned}
\xi & =r \cos \varphi \sin \theta \\
\eta & =r \sin \varphi \sin \theta \\
\zeta & =r \cos \theta
\end{aligned}
$$

into (38) and set the derivatives $\partial U / \partial \varphi$ and $\partial U / \partial \theta$ equal to zero.

This results in the following pair of equations in $\varphi$ and $\cot \theta$;

$$
\begin{array}{r}
a_{1} \cos ^{k} \varphi \sin \varphi+a_{2}(\cos \varphi-\sin \varphi)^{k}(\cos \varphi+\sin \varphi) \\
=a_{3}(\sin \varphi-\cot \theta)^{k} \cos \varphi \\
a_{1} \cos ^{k+1} \varphi+a_{2}(\cos \varphi-\sin \varphi)^{k+1} \\
=a_{4}-a_{3}(\sin \varphi-\cot \theta)^{k}(\sin \varphi+1 / \cot \theta)
\end{array}
$$

Evidently, they are sufficient to determine the required angles.

It is instructive to reduce these equations to the linear case and equal springs. In that case, the foregoing equations become

$$
\begin{gathered}
\cos ^{2} \varphi-\sin ^{2} \varphi=-\cos \varphi \cot \theta \\
2 \cos \varphi=(1 / \cot \theta)-\cot \theta
\end{gathered}
$$

This pair of equations has the following roots:

$$
\begin{array}{ll}
1 \text { mode: } \varphi \cong 54^{\circ} 45^{\prime}, \theta=60^{\circ} \\
2 \text { mode: } \varphi=0, & \theta=135^{\circ} \\
3 \text { mode: } \varphi \cong 305^{\circ} 15^{\prime}, \theta=60^{\circ}
\end{array}
$$

The eigenvectors corresponding to these roots are shown in Fig. 4 . 


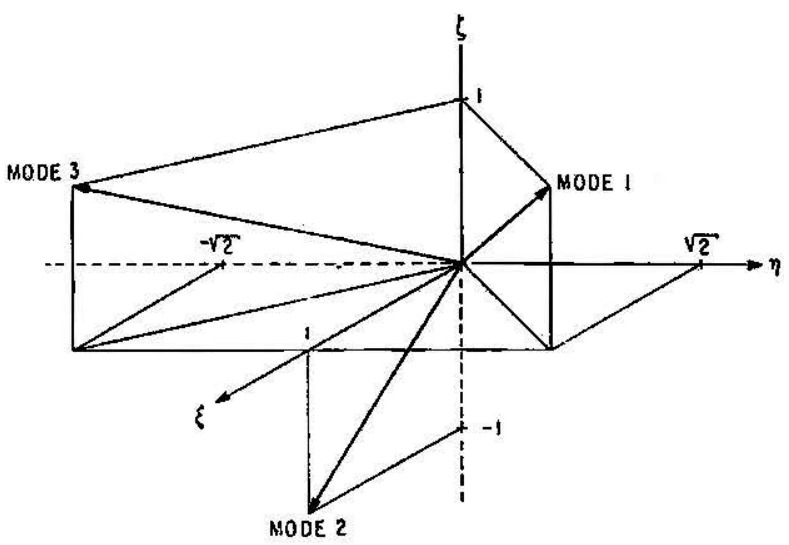

Fig. 4

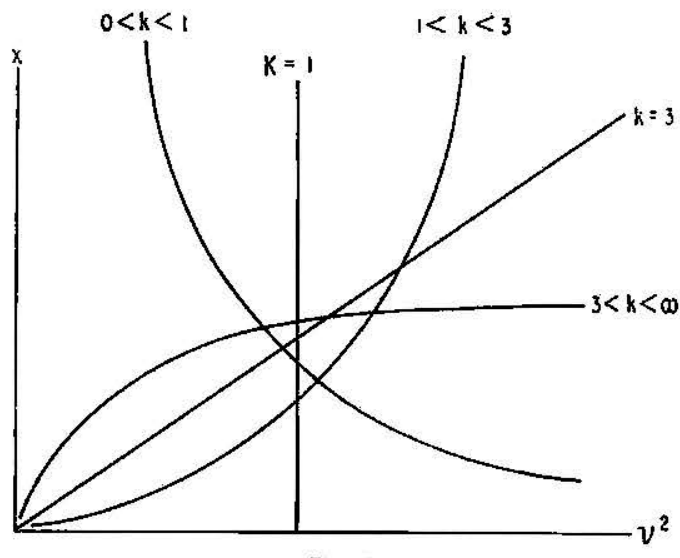

Fig. 5
The roots of the transcendental equation yield the following wellknown results [5] (as is evident from Fig. 4):

$$
\begin{array}{lll}
1 \text { mode: } & \eta=2^{1 / 2 \xi}, & \xi=\xi, \\
2 \text { mode: } & \eta=0, & \zeta=-\xi, \\
3 \text { mode: } & \eta=-2^{1 / 2} \xi, & \zeta=\xi
\end{array}
$$

In the nonlinear homogeneous case, the solution of the transcendental equations will yield the values of the constants $c$ in

$$
\eta=c_{\eta} \xi, \quad \zeta=c_{\zeta r} \xi, \quad r=1,2, \ldots
$$

and the largest value of $r$ is equal to the number of modes. In general, one may expect as many sets of constants as there are degrees of freedom (i.e., here three), but the existence of superabundant modes [3] is not excluded. In all that follows, it will be assumed that the transcendental equations (40) have been solved, and that the constants in (41) are known.

\section{The Eigenvalues}

If the first of (41) is sulsstituted in the first equation of motion, one finds [2] (in view of the assumption that the three masses are equal)

$$
\ddot{\xi}=-\left[a_{1}+a_{2}\left(1-c_{\eta r}\right)^{k}\right] \xi ; r=1,2, \ldots
$$

If the eigenvalues are denoted by $\omega_{r},(r=1,2, \ldots),(42)$ is integrated over one period under the initial conditions $\xi(0)=\chi$, $\xi(0)=0$, and use is made of the nomenclature $\omega^{2} / a_{2}=\nu^{2}$, $a_{1} / a_{2}=\alpha$, it has been shown [3] that

$$
\nu_{r}^{2}=\left[\frac{\Gamma\left(\frac{1}{k+1}+\frac{1}{2}\right)}{\Gamma\left(\frac{1}{k+1}\right)}\right]^{2} \pi \frac{k+1}{2}\left[\alpha+\left(1-c_{\eta r}\right)^{k}\right] \chi^{k-1}
$$

Before discussing this equation, it should be remarked that our entire discussion of the homogeneous system remains valid for noninteger, positive values of $k$ if provisions are made for the odd character of the spring forces. This can be done, for instance, by replacing terms of the sort $\xi^{k}$ in the equations of motion by $\xi|\xi|^{k-1}$ and terms like $\left(\xi_{i-1}-\xi_{i}\right)^{k+1}$ in the potential functions by $\left|\xi_{i-1}-\xi_{i}\right|^{k+1}$. The reason for the extended validity of our discussion is that, with these provisions, the equipotential surfaces remain symmetric with respect to the origin.

Equation (43) has been discussed heretofore [3]; it is the equation of the frequency-amplitude relations of free normal vibrations, called by Klotter [6] the backbones. A number of familiar properties stand out. For instance, in the linear problem, $(k=1)$, the frequency of free vibrstions is independent of the amplitude, while, in the nonlinear case, the frequency of free vibrations does depend on the amplitude.

When the spring is hard $4(k>1)$, the amplitude increases with the frequency; when the spring is $\operatorname{soft}^{4}(0<k<1)$, the amplitude decreases when the frequency increases. These and other properties of the backbone curves, easily deducible from (43), are illustrated in Fig. 5.

\section{Maximum-Minimum Properties}

It is well known [7] that the eigenvalues of linear problems possess maximum-minimum properties. Moreover, it has been shown [2] that the modal relations of the (linear or nonlinear) two-degree-of-freedom problem also have such properties. We would like now to show that this property also holds for $n$ degrees of freedom.

To do this, we focus attention on the geodesics in the space whose metric is

$$
d \sigma^{2}=\left(U_{0}+U\right) \sum_{i=1,2, \ldots}^{n} d \xi_{i}{ }^{2}
$$

Clearly, all are solutions of the dynamical problem. Suppose we seek the geodesics between the origin of this $n$-space and a point which is movable on the bounding surface $U=-U_{0}$. This movable-end-point problem produces not only the Euler equations (30) (and thus solutions to the dynamical problem), but also transversality conditions. But these transversality conditions state [8] that the geodesics must intersect the bounding surface orthogonally. Therefore they are the modal relations.

In other words, the modal relations are the shortest and the longest of the lines of minimum length (geodesics) in the $n$-space between any point on the bounding surface and the origin, where the space is defined by the metric (28).

\section{Acknowledgments}

Financial aid from the National Science Foundation which has supported this investigation is gratefully acknowledged. The author wishes particularly to express his appreciation to the Director of the Institut de Chronométrie et de Micromécanique of the Faculté des Sciences at Besançon, Mr. P. Mesnage, who has kindly put the facilities of the Institute at his disposal for the completion of this study.

In hard springs, the spring stiffness (i.e., the slope of the spring force) increases with increasing defleotion, in soft springs, it decreases with increasing deffection. 


\section{References}

1 R. M. Rosenberg and C. P. Atkinson, "On the Natural Modes and Their Stability in Nonlinear Two-Degree-of-Freedom Systems," Journat of Applited Mrohanics, vol. 26, Trans. ASME, Series E, vol. 81,1959 , pp. $377-385$.

2 R. M. Rosenberg, "Normal Modes of Nonlinear Dual-Mode Systems," Jodrnal of Applied Menanames, vol. 27, Trans. ASME, vol, 82, Series E, June, 1960, pp. 263-268.

3 R. M. Rosenberg, "On Normal Vibrations of a General Class of Nonlinear Dual-Mode Systems," Journal of Applied Mechanrcs, vol. 28, Trans. ASME, vol. 83, Series E, June, 1961, pp. 275-283.

4 G. Darboux, "Leçons sur la Théorie Générale des Surfaces," Gauthier-Villars et Fils, Paris, France, vol. 2, 1889, p. 453.
5 J. P. Den Hartog, "Mechanical Vibrations," MoGraw-Hill Book Company, Inc., New York, N. Y., fourth edition, 1956, p. 126.

6 K. Klotter, "Steady State Vibrations in Systems Having Arbitrary Restoring and Arbitrary Damping Forces," Proceedings of the Second U.S. National Congress of Applied Mechanics, University of Michigan, Ann Arbor, Mich., 1954, pp. 69-78.

7 R. Courant and D. Hilbert, "Methods of Mathematical Physics," vol. 1, Interscience Publishers, Inc., New York, N.,Y., 1953, pp. 405-407.

8 O. Bolza, "Vorlesungen úber Varationsrechnung," Koehler und Amelang, Leipzig, Germany, revised and much enlarged, 1949, pp. 553-691. 